\title{
Effect of powdered activated carbon particle size on the flow field in membrane filtration system
}

\author{
Anhu Dang ${ }^{2}$, Xingfei Gou ${ }^{1,2^{*}}$, Hui Jia ${ }^{1,2}$, Yi Wu$^{2}$ \\ ${ }^{1}$ State Key Laboratory of Membrane filtration and Processes, Tianjin Polytechnic University, Tianjin \\ 300387, China; \\ ${ }^{2}$ School of Environmental and Chemical Engineering, Tianjin Polytechnic University, Tianjin 300387, \\ China. \\ *feifei_0630@sina.com
}

Corresponding Author: Xingfei Gou

Keywords: flow field, membrane filtration, powdered activated carbon, shear stress.

\begin{abstract}
The effect of powdered activated carbon (PAC) particle size in the range of 0.09 0.18mm on the flow field in membrane filtration process was investigated. The SEM images of membrane surface showed that it might cause damage on the membrane surface in membrane filtration system by the addition of PAC. Exceptional instantaneous velocity was introduced to describe flow field variation. Under a submerged membrane filtration mode, the influence of PAC addition on flow field and shear stress was more pronounced than that of the system without PAC addition. Compared with the flow field and membrane surface shear stress of other PAC systems, the PAC with particle size of $0.15 \mathrm{~mm}$ accompanying with aeration rate of $5 \mathrm{~L} / \mathrm{min}$ was the optimal condition for the slight damage and good cleaning to membrane fiber.
\end{abstract}

\section{Introduction}

It is considered as one of the most effective methods to control membrane fouling that adding PAC in the filter tank. Study by Lin et al.[6] found that dosing PAC could change the sludge characteristics, and improve system performance. Study by Sun et al.[7] showed that the PAC-MBR process had an obvious advantage for the removal of total phosphorus (TP). Above works were largely confined to improving the properties of the mixture by adding PAC. However, few studies have been reported about the effect of PAC on the flow field distribution.

As aeration drives the activated carbon flow irregularly, dosing carrier in the filtration system not only changes the nature of the mixture, but also affects the hydraulic fluid flow conditions.

In this study, the flow field and membrane surface shear stress distribution in the PAC-membrane filtration process has been evaluated. The study aimed to optimize aeration intensity and the particle size of PAC, coming up with the optimal combination of PAC with the membrane filtration system.

\section{Materials and methods}

\subsection{Bench-scale apparatus}

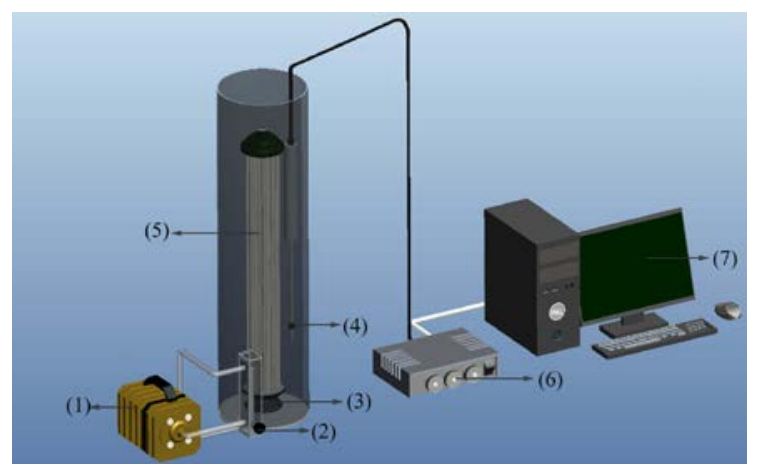

Fig.1. Illustration of the bench-scale apparatus to detect velocity: (1) air pump, (2) flowmeter, (3) air diffuser, (4) velocity measurement probe, (5) membrane module, (6) signal converter, and (7) computer. 
A bench-scale apparatus is shown in Fig.1 The apparatus consists of membrane filtration system, membrane module, aeration system, velocity-measuring system, and computer. Membrane filtration tank $(0.07 \mathrm{~m} \times 1.2 \mathrm{~m}$ by radius and height) is an cylindrical polymethyl methacrylate composition. The membrane module $(0.04 \mathrm{~m} \times 0.8 \mathrm{~m}$ by radius and height) is made of PVDF hollow fiber membrane (Tianjin Motimo Membrane Technology Co.,Ltd., China). And the liquid used is deionized water, liquid level is $1.0 \mathrm{~m}$. The diffuser installed at the bottom of the reactor tank with the distance of $0.1 \mathrm{~m}$ from the membrane module.

\subsection{Experimental conditions}

PAC was screened by pharmacopoeia seperator (Dadi, ShangYu, China) having different particle sizes of $0.09,0.125,0.15$, and $0.18 \mathrm{~mm}$. According to previous researches, the addition of PAC was $2 \mathrm{~g} / \mathrm{L}[18,19,20]$. Aeration intensity were controlled at 3, 5, and 7L/min, respectively. Experiment was conducted in seven cross sections at $10 \mathrm{~cm}, 20 \mathrm{~cm}, 30 \mathrm{~cm}, 40 \mathrm{~cm}, 50 \mathrm{~cm}, 60 \mathrm{~cm}$, and $70 \mathrm{~cm}$ apart from the air diffuser. On each section, four test points were selected. Thus, there is a total of 28 test points. The data were gathered for 2 min continuously with the collection frequency of $50 \mathrm{~Hz}$ at each test point. The exceptional instantaneous velocity percentage (X\%) was calculated by:

$$
X \%=\frac{N_{1}}{N_{0}} \times 100 \%
$$

Where $N_{1}$ is the number of exceptional instantaneous velocity which is greater than $10 \mathrm{~m} / \mathrm{s}, N_{0}$ is the number of all the velocity gathered within special time.

\section{Results and discussion}

\subsection{The exceptional instantaneous velocity percentage analysis}

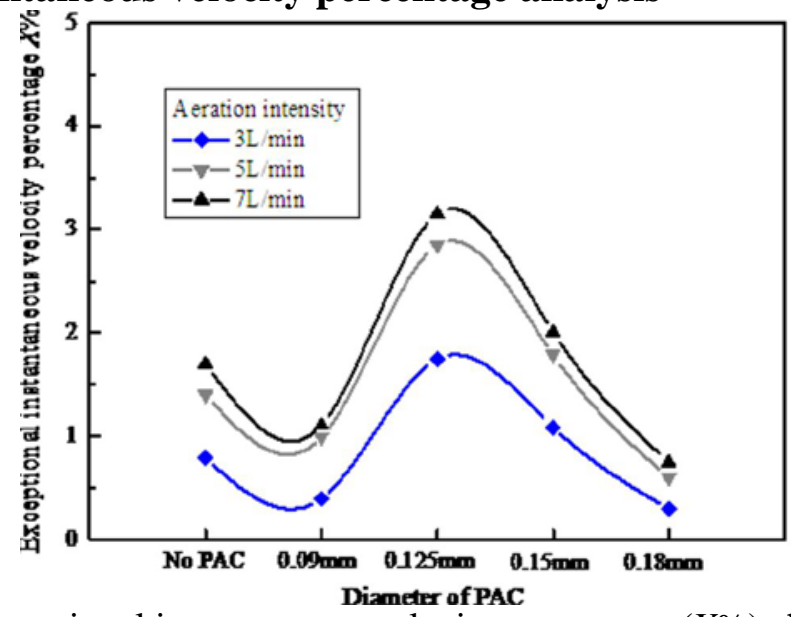

Fig. 2 Exceptional instantaneous velocity percentage $(X \%)$ change rule

Fig.2 illustrates that with the increase of aeration rate, variations of average velocity in the reactor aggrandized, when aeration rate was $3 \mathrm{~L} / \mathrm{min}$, while exceptional instantaneous velocity percentage was minimum, its overall average velocity was also smaller. However, when aeration rate was $7 \mathrm{~L} / \mathrm{min}$, the percentage of exceptional instantaneous velocity got maximum. Exceptional instantaneous velocity drives PAC to flow fleetly, and a sudden increase of intense physical exercise of PAC can cause a certain degree of damage to membrane fiber. In comparison, the aeration rate of $5 \mathrm{~L} / \mathrm{min}$ was the optimal aeration in this study. 


\subsection{The velocity field analysis}

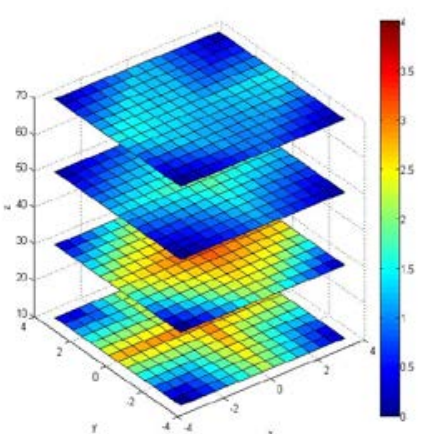

(a)

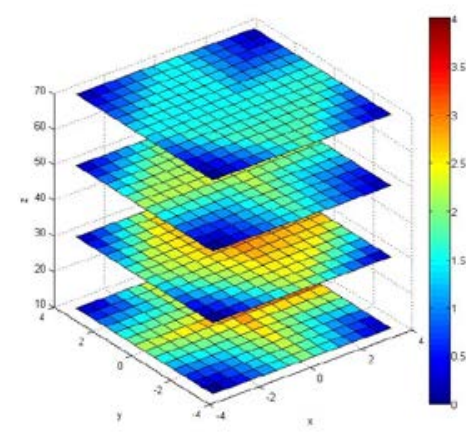

(b)

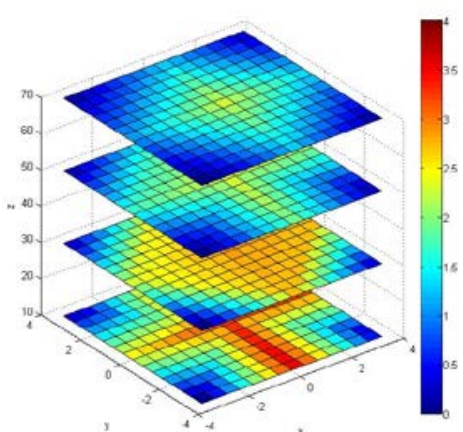

(c)

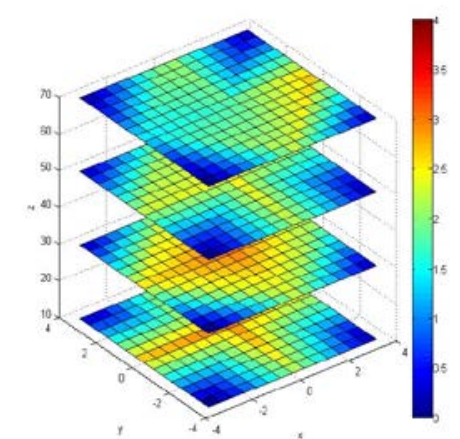

(d)

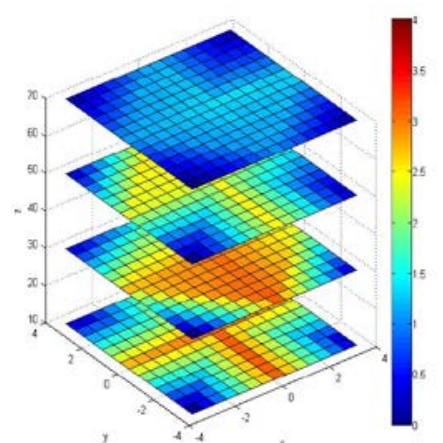

(e)

Fig. 3 The distribution of velocity field with different particle sizes of activated carbon when the aeration rate was 5L/min: (a) no PAC, (b) 0.09mm PAC, (c) $0.125 \mathrm{~mm}$ PAC, (d) $0.15 \mathrm{~mm}$ PAC, (e) $0.18 \mathrm{~mm}$ PAC.

Fig.3a showed the velocity field of the system without PAC, where were significant high velocity areas at the bottom and low velocity areas at the upper side in the reaction system. Fig.3b, c showed the velocity field of the system with PAC diameter of $0.09 \mathrm{~mm}$ and $0.125 \mathrm{~mm}$, the high velocity areas gathered in the bottom, while velocity at upper side was small. In Fig.3e, the velocity field was small and uneven for the larger PAC with diameter of $0.18 \mathrm{~mm}$.

Large bubbles induced by aeration were separated into numerous small bubbles with the existence of PAC, and the small bubbles distributed uniformly on the surface of membrane. Large bubbles make intensive shear forces which can damage the membrane fiber and small bubbles make the weak one which is advantageous to the comprehensive scouring of the membrane surface. The activated carbon diameter of $0.15 \mathrm{~mm}$ (Fig.3d) was the optimal one, where the velocity field was well-distributed relatively and gathered near the membrane surface.

\subsection{The average velocity and average membrane shear stress analysis}

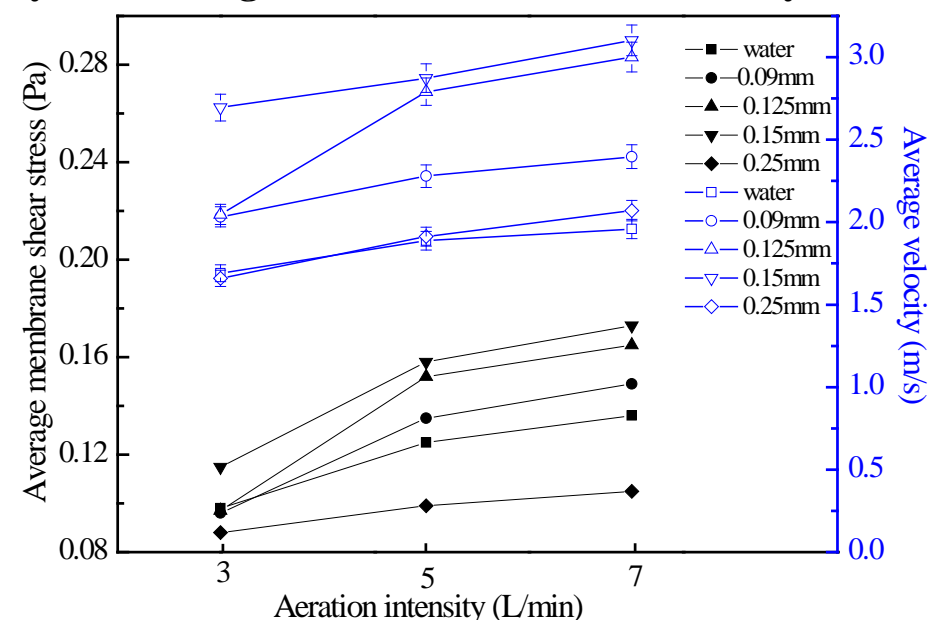

Fig. 4 The variations of average velocity and membrane surface shear stress

In Fig.4, the variation trend of the average membrane surface shear stress was in keeping with that of average velocity. With the increase of aeration rate from $3 \mathrm{~L} / \mathrm{min}$ to $7 \mathrm{~L} / \mathrm{min}$, average velocity 
and shear stress in the five different system appeared to be stabilized after the first increased trend. When the aeration rate was $5 \mathrm{~L} / \mathrm{min}$, the moderate velocity appeared with PAC of $0.15 \mathrm{~mm}$ in particle size, which could drive the activated carbon to flow fleetly, reduce the damage on the membrane.

\section{Conclusions}

The SEM images of the membrane surface showed that PAC addition might damage the membrane fibers in membrane filtration system. Exceptional instantaneous velocity percentage $(\mathrm{X} \%)$ indicated the aeration rate of $5 \mathrm{~L} / \mathrm{min}$ was the optimal aeration in this study. $0.15 \mathrm{~mm}$ PAC system had smaller average flow velocity and exceptional instantaneous velocity percentage (X\%) than that of $0.125 \mathrm{~mm}$ PAC system, followed by the other systems. Compared with the other PAC systems, the velocity field was well-distributed and gathered near the membrane surface of $0.15 \mathrm{~mm}$ PAC system. And the average membrane surface shear stress was appropriate, which was in keeping with the variation of average velocity. This could scour membrane surface powerfully, and minish the injury on membrane fiber, so as to mitigate membrane fouling and prolong the life of the membrane. Thus, the PAC with particle size of $0.15 \mathrm{~mm}$ accompanying with aeration rate of $5 \mathrm{~L} / \mathrm{min}$ was the optimal condition in this bench-scale system.

\section{Acknowledgement}

This study is financially supported by the National Natural Science Foundation of China (No.51378349) and Program for Changjiang Scholars and Innovative Research Team in University (IRT13084) and China Postdoctoral Science Foundation (2013M541184).

\section{References}

[1] H.J. Lin, X.F. Lu, W.L. Wang, The study of the impact of dosing PAC on membrane bioreactor processing municipal secondary effluent, Membrane separation technology applied in the oil and chemical industry conference papers, 2006, 84-85.

[2] Y.Y. Sun, Z.S. Bo, Z.G. Si, et al. Study on MBR and PAC-MBR for micro polluted source water phosphorus removal effect, North Environment, 29 (2013) 134-135.

[3] P.R. Berube, G. Afonso, F. Taghipour, et al. Quantifying the shear at the surface of submerged hollow fiber membranes, Journal of Membrane Science, 279 (2006) 495-505.

[4] C.C.V. Chan, P.R. Berube, E.R. Hall, Shear profiles inside gas sparged submerged hollow fiber membrane modules, Journal of Membrane Science, 297 (2007) 495-505.

[5] V.E. Nakoryakov, O.N. Kashinsky, B.K. Kozmenko, Experimental study of gas-liquid slug flow in a small diameter vertical pipe, International Journal of Multiphase Flow, 12 (1986) 337-355.

[6] C. Gaucher, P. Legentilhomme, P. Jaouen, et al. Hydrodynamics study in a plane ultrafiltration module using an electrochemical method and particle image velocimetry visualization, Experiments in Fluids, 32 (2002) 283-293.

[7]Fang, H.H.P.; Shi, X.; Zhang, T. Effect of activated carbon on fouling of activated sludge filtration. Desalination 189 (2006) 193-199.

[8]Ng, C.A., Sun, D., Fane, A.G. Operation of membrane bioreactor with powdered activated carbon addition. Sep. Sci. Technol. 41 (2008) 1447-1466.

[9]Torretta, V., Urbini G., P. Viotti, Luciano A., Mancini G, Effect of powered activated carbon to reduce fouling in membrane bioreactors: a sustainable solution. Case study, Sustainability, 5 (2013) 1501-1509. 